\begin{tabular}{c} 
Volume and Issues Obtainable at Center for Sustainability Research and Consultancy \\
Journal of Accounting and Finance in Emerging Economies \\
ISSN: 2519-0318 ISSN (E) 2518-8488 \\
Volume 6: Issue 3 September 2020 \\
CSRC \\
Journal homepage: www.publishing.globalcsrc.org/jafee \\
\hline
\end{tabular}

\title{
Co-movement between Sukuk, Conventional Bond and Islamic Stock Markets under Bullish and Bearish Market Conditions: An Application of Quantile-on-Quantile Regression
}

\author{
${ }^{1}$ Fatima Farooq, ${ }^{2}$ Muhammad Saeed Meo, ${ }^{3}$ Sajid Ali, ${ }^{4}$ Usman Rasheed \\ ${ }^{1}$ Assistant Professor, School of Economics, Bahauddin Zakariya University Multan, Pakistan, \\ fatimafarooq@bzu.edu.pk \\ ${ }^{2}$ The Superior College Lahore Pakistan, Saeedk8khan@ gmail.com \\ ${ }^{3}$ Faculty of Economics and Management, Universiti Putra, Seri Kembangan, Malaysia, sajidali1136@gmail.com \\ ${ }^{4}$ The Superior College Lahore Pakistan, usman.rasheed840@ gmail.com
}

\begin{abstract}
ARTICLE DETAILS
\section{History}

Revised format: Aug 2020

Available Online: September

2020

Keywords

$Q Q$ regression, Sukuk market, Conventional bond market, Islamic stock market

JEL Classification:

M40, M41

\section{ABSTRACT}

This study explores the asymmetric co-movement between conventional bonds market, sukuk market and Islamic stock markets of top ten Islamic economies. The study used daily data ranging from $1^{\text {st }}$ January 2008 to $31^{\text {st }}$ December 2019. However, for the dependency structure, we used Quantileon-Quantile (QQ) method, which captures the dependence between the entire distributions of financial assets and uncovers some nuance features of the relationship. The empirical findings show that under the stress condition (bearish condition), both bonds and stocks markets negatively commove. However, in the bullish market condition, these markets show week positive correlation/ co-movement in all the sample economies. The findings also confirmed that under the bearish condition, a mild negative correlation exists between sukuk and Islamic stock markets except for Malaysia. However, in the bullish market condition, sukuk markets and stock markets show strong positive correlation in all sample economies. Furthermore, study also enriches quantiles estimation by using quantiles granger causality approach. The findings show that the conventional bond market, sukuk market and Islamic stock market granger cause to each other on all the quantiles (in bullish or bearish market conditions).
\end{abstract}

\section{OPEN ACCESS}

(C) 2020 The authors, under a Creative Commons Attribution-

NonCommercial 4.0

Corresponding author's email address: fatimafarooq@bzu.edu.pk

Recommended citation: Farooq, F., Meo, M. S., Ali, S. \& Rasheed, U. (2020). Co-movement between Sukuk, Conventional Bond and Islamic Stock Markets under Bullish and Bearish Market Conditions: An Application of Quantile-on-Quantile Regression. Journal of Accounting and Finance in Emerging Economies, 6(3), 839-856

\section{Introduction}

The nexus between the Sukuk, conventional bond and Islamic stock markets is of particular interest for both portfolio managers and policymakers as it has important repercussions for asset allocation and risk management strategies. They reflect and encapsulate investors' behavior and responses to changing market conditions as well as their forward-looking perspectives or the economy (Nasir and Farooq, 2017). Hence, not surprisingly, the determinants and the dynamics that govern the relationship between the two markets have been a popular theme in the relevant 
literature (Ahmed and Elsayed, 2019; Duqi and Al-Tamimi, 2019).

Islamic Stocks index and Conventional bonds market have different risk-return characteristics and are of great interest for traders, portfolio managers as well as policymakers. Many scholars have been involved in plentiful theoretical and empirical studies to provide a better understanding of the interplay between these two classes of financial assets (Shahzad, Raza, Shahzad and Ali, 2017; Duqi and Al-Tamimi, 2019). The research is motivated by several theoretical arguments of the Islamic stock index-conventional bond market interplay related to the price discovery information process, contagion effects, capital market efficiency, volatility spillovers, portfolio' allocations and optimal hedging strategies (Ahmed and Elsayed, 2019).

In financial markets, sharia-compliant Islamic stocks index and sukuk market are the two main assets classes to design investment portfolios. On the academic side, it is very important to answer the question of whether Islamic bonds (sukuk market) have the same behavior as conventional bonds market in terms of co-evolution. In this vein, many counterarguments are identified in the Islamic finance literature, but no empirical consensus is reached. For Islamic portfolio managers, it is very important to comprehend the dynamic linkage between the two financial markets since they can provide useful implications in terms of creating effective hedging strategies and informational efficiency. It can help to understand how Islamic assets are co-reacting to the arrival of new information and their degree of persistence to innovations through time (Ahmed and Elsayed, 2019). For policymakers, it is well recognized that the co-movement between these markets is essential in the sense that it may be expedient for assessing policy effectiveness.

Currently, the related literature is extremely profuse and mainly concerned with developed and emerging capital markets, however, no particular attention is paid to analyze stock-conventional bond interplay in the Islamic financial markets. The global events that have shaken the world economy are now more insistent and frequent in large developed and emerging economies worldwide. The synchronization of economic sectors and sustainable international financial integration is considered as the essentials for the market turbulences (Coeurdacier, Rey, and Winant, 2020) Modeling the dependence structure between financial assets is of immense importance to construct an optimal portfolio. The current study will cover the existing gap by providing co-movement between sukuk, conventional bonds and Islamic stock markets under different market conditions.

\section{Literature Review}

In this section, we are giving a brief review of some previous studies which analyzes the relationship between conventional bond, sukuk and Islamic stock.

Naifar (2016) investigated the dependence structure between Islamic bond (sukuk) and stock market in Saudi Arabia by using Archimedean copula models. The findings revealed that the sukuk yield exhibited significant and symmetric dependence with stock market returns. Ahmed and Elsayed (2019) analyzed the dynamic interdependencies among bonds, sukuk markets, conventional stock and Islamic stock in Malaysia. It was found that conventional bond and stock markets were the main net transmitters of spillovers from other markets, while sukuk market was a net receipt of return shocks from Islamic, conventional and bond markets. In another study, Shahzad et al. (2017) examined the association of gold and conventional bonds with stock market in USA, UK, Japan, Canada and Germany by using the novel vector autoregressive and found that gold was a strong hedge and diversifier for the stock portfolio except when both market are under stress. Nasir and Farooq (2017) analyzed the value of risk of conventional bonds and sukuk in Pakistan and revealed that sukuk were less risky and more stable instrument as compared to conventional bonds. Shahzad et al. (2020) examined the safe haven property of gold for stock and conventional bond markets of G-7 countries by using the cross quantilogram method and observed that gold did not act as a safe haven for stock and conventional bond markets. In another study, Mcmillan (2019) examined the behavior of stock and conventional bond and observed that stock and conventional bond return had significant predictive power to other country stock and conventional bond return. 
It is cleared from the review of literature that previous studies are mainly concerned with developed and emerging capital markets, however, no particular attention is paid to analyze the co-movement between conventional bond market, sukuk and Islamic stock in different market conditions (i.e. bulish and bearish conditions) as these markets interplay in the financial markets. Co-movement between conventional bonds market, sukuk market and Islamic Stocks index is the critical issue for portfolio's designing and hedging strategies for investors who have hugely different time investment horizons. So in this study, we analyze the relationship between these assets from a different angle under different market conditions in the top 10 Islamic stock markets of the world. No previous study has analyzed the asymmetric or non-linear relationship between these variables at both bottom and upper quantiles of the distribution of data by applying a new approach "Quantile-on-Quantile".

\section{Data and Methodology}

\section{Variables and Data Sources}

This study examines co-movement between conventional bonds, sukuk and Islamic stocks under bearish and bullish market conditions in top 10 Islamic stock markets ${ }^{1}$ based on daily data ranging from $1^{\text {st }}$ January 2008 to $31^{\text {st }}$ December 2019 and extracted from DataStream database. Following the studies of Naifer (2016) and Nasir and Farooq (2017), we have taken Islamic stock as the dependent variable while conventional bond and sukuk market as independent variables. We use Quantile-on-Quantile (QQ) approach, which captures the dependence between the entire distributions of financial assets and uncovers some nuance features of the relationship.

\section{Quantile Autoregressive Unit Root Test}

The stationary properties of the time series data at each quantile and on the conditional mean is checked by Quantile Auto-Regressive (QAR) proposed by Koenker and Xiao (2004). The basic characteristics of QAR model are generalized by Galvao (2009) by considering linear time trends and covariates in model. The numerous tenacity parameters $(\widehat{\alpha})$ for each quantile of the conditional distribution of $\mathrm{X}_{\mathrm{t}}$ is estimated and t-statistics for various quantiles $\tau \epsilon T$ are obtained to test the null hypothesis $H_{0}: \alpha(\tau)=1$.

\section{Quantile Cointegration Test}

The novelty of the current study lies in its endeavor to analyze the systematic influence of various frequencies of stock market on the shape, location and scale of the Islamic stock index by applying Quantile Cointegration Test of Xiao (2009). To deal with the endogeneity problem in cointegration model, Xiao (2009) decomposed the cointegration equation errorrs into pure innovation component and lead-lag terms. Furthermore, the Quantile Cointegration Test of Xiao (2009) is the extended form of the cointegration tset proposed by Engle and Granger (1987).

Xiao (2009) took the null hypothesis as $H_{0}: \beta(\tau)=\beta$ over all the quantiles. A rule of the absolute value of the difference $\widehat{V}_{n}(\tau)=(\hat{\beta}(\tau)-\hat{\beta})$ is proposed as a test statistic under null hypothesis. The test statistic $\sup _{\tau}\left|\widehat{V}_{n}(\tau)\right|$ across all the quantiles distribution is employed. Following Xiao (2009), this study applies 1000 Monte Carlo simulations to calculate the critical values of $\sup _{\tau}\left|\widehat{V}_{n}(\tau)\right|$.

\section{Quantile on Quantile Approach}

Most of previous studies had analyzed the association between two variables by employing linear regression and then move to the Quantile Regression Approach (QRA) of Koenker and Bassett (1978). However, the QRA framework is unable to capture the entire dependency structure between the variables. Although the QRA framework can consider heterogeneity at various points of the conditional distribution, it cannot deal with the the nature of uncertainty which may also affect the relationship between independent and dependent variables. Owing to this lacuna of QRA, Sim and Zhou (2015) introduced a novel approach named "Quantile-on-Quantile (QQ)" which can excellently deal with all these problems.

The QQ methodology would help in capturing the variations in the association between the variables at each point of their conditional distribution and provides a complete and clear picture of the dependency relationship. The study

\footnotetext{
${ }^{1}$ Bahrain, Brunei, Kuwait, Libya, Malaysia, Oman, Qatar, Saudi Arabia, Turkey and United Arab Emirates.
} 
employs the QQ framework by selecting some uncertainty quantiles and by analyzing the local effect of conventional bonds market (CBM) and sukuk market (SM). In recent years, a remarkable work was done by using QQ approach to estimate the association between the variables at different bottom and top quantiles (Sharif, Afshan, and Qureshi, 2019; Chang et al., 2020; Sharif et al., 2020).

Suppose, ISI represents the Islamic stock index of the country at time $t$. CBM and SM is the conventional bond market and sukuk market at time $t$, respectively. $\mu_{\mathrm{t}} i s$ the quantile residual term. These quantile regression methods help to analyze the empirical effects of the conventional bond market and sukuk market across the different quantiles of Islamic stock market.

$$
\begin{aligned}
& I S I_{t}=\beta^{\theta}\left(C B M_{t}\right)+\mu_{t}^{\theta} \\
& I S I_{t}=\beta^{\theta}\left(S M_{t}\right)+\mu_{t}^{\theta}
\end{aligned}
$$

This regression technique is flexible because it evaluates the functional dependence between Islamic stock index (ISI), conventional bond and sukuk market in the top ten Islamic countries. The main benefit of the specification is its flexibility because no previous hypothesis exists regarding the functional relationship between sukuk market and stock market growth. Eq.(1) is examined the neighborhood of Islamic stock index employing local linear regression.

$$
\begin{aligned}
& \beta_{0}(\mathrm{ISI})=\beta_{0}(\mathrm{CBM})+\beta_{0}\left(\mathrm{CBM}^{\mathrm{t}}\right)\left(\mathrm{CBM}_{\mathrm{t}^{-}} \mathrm{CBM}^{\mathrm{t}}\right) \\
& \beta_{0}(\mathrm{ISI})=\beta_{0}(\mathrm{SM})+\beta_{0}\left(\mathrm{SM}^{\mathrm{t}}\right)\left(\mathrm{SM}_{\mathrm{t}^{-}} \mathrm{SM}^{\mathrm{t}}\right)
\end{aligned}
$$

$\beta$ o is partial derivative of $\beta^{\theta}\left(C B M_{t}\right)$ with respect to $\left(S M_{t}\right)$, also called marginal effect or response. The parameters $\beta_{0}(\mathrm{CBM})$ and $\beta_{0}(\mathrm{SM})$ are the doubly index in $\theta$ and , that give $\beta_{0}\left(C B M_{t}\right)$ is function of $\theta$ and $\beta_{0}(\mathrm{SM})$ is function of $\tau$, and additional $\beta_{0}(\mathrm{SM})$ can be renamed as $\beta_{0}(\theta, \tau)$. Accordingly, the revised forms of eq 3 and 4 can be written as follows:

$$
\begin{aligned}
& \beta_{0}(\mathrm{ISI})=\beta_{0}(\theta, \tau)+\beta_{0}(\theta, \tau)+\left(\mathrm{CBM}_{\mathrm{t}}-\mathrm{CBM}^{\mathrm{t}}\right) \\
& \beta_{0}(\mathrm{ISI})=\beta_{0}(\theta, \tau)+\beta_{0}(\theta, \tau)+\left(\mathrm{SM}_{\mathrm{t}}-\mathrm{SM}^{\mathrm{t}}\right)
\end{aligned}
$$

By substituting Eq.(5) and Eq.(6) in Eq. (1), the following equations of QQ methodology are obtained:

$$
I S I_{t}=\underbrace{\beta_{0}(\theta, \tau)+\beta_{1}(\theta, \tau)(C B M-C B M)}_{*}+u_{t}^{\theta} \quad \text { Eq.(7) }
$$$$
I S I_{t}=\underbrace{\beta_{0}(\theta, \tau)+\beta_{1}(\theta, \tau)\left(S M_{t}-S M^{\tau}\right)}_{*}+u_{t}^{\theta}
$$

Equations 7 and 8 show the functional form of QQ methodology. The part (*) of Eq.7 and 8 is the $\theta$ th conditional quantile of Islamic stock Index. Eq. (7) reflects the relationship between $\theta$ th quanlite of the conventional bond market and the $\tau$ th quanlite of islamic stock index. On the other hand, Eq. (8) shows the association between $\theta$ th quanlite of sukuk market and the $\tau$ th quanlite of Islamic stock index. The quantile relationship between conventional bond market, sukuk market and Islamic stock index is truly established due to the parameters $\beta_{0}$ and $\beta_{1}$, which are doubly indexed in $\theta$ and $\tau$. These parameters vary due to different values of $\theta^{\text {th }}$ quantiles of conventional bond and stock market. In Eq. (7) and (8), we estimate the overall dependance structure between co-movement of sukuk market, conventional bond market and Islamic stocks market.

\section{Results and Discussion}

Table1 exhibits the result of quantile unit root test. The stationary test documents the presence of unit root at the level for conventional bonds market, sukuk market and Islamic stock index for the different conditional distribution of quantiles. The result of the unit root test confirms that the entire variables are showing non-stationary behavior at level. 
ISI Journal of Accounting and Finance in Emerging Economies

CBM

Vol. 6, ISI 3, September 2820

ISI

t-stats

\begin{tabular}{|c|c|c|c|c|c|c|c|c|c|c|c|c|c|c|c|c|c|c|c|c|}
\hline \multirow[b]{2}{*}{0.05} & \multicolumn{3}{|c|}{ Bahrain } & \multicolumn{3}{|c|}{ Brunei } & \multicolumn{7}{|c|}{ Kuwait } & \multicolumn{2}{|c|}{ Libya } & \multicolumn{4}{|c|}{ Malaysia } & \multirow[b]{2}{*}{0.674} \\
\hline & 1.001 & 0.058 & 0.971 & -0.128 & 0.922 & -1.559 & 0.988 & -0.292 & 0.972 & -0.583 & 0.992 & -0.114 & 0.980 & -0.369 & 0.978 & -0.250 & 0.973 & -1.748 & 1.001 & \\
\hline 0.10 & 1.001 & 0.174 & 0.979 & -0.166 & 1.010 & 0.379 & 0.989 & -0.354 & 0.979 & -2.027 & 1.001 & 0.031 & 0.985 & -1.619 & 0.984 & -0.824 & 0.986 & -2.446 & 1.000 & 0.904 \\
\hline 0.15 & 0.998 & -1.298 & 0.972 & -0.865 & 1.000 & 0.012 & 0.988 & -2.252 & 0.980 & -2.383 & 0.996 & -0.375 & 0.990 & -1.950 & 0.995 & -0.362 & 0.986 & -2.044 & 1.000 & 1.274 \\
\hline 0.20 & 0.998 & -1.343 & 0.968 & -2.582 & 0.991 & -1.007 & 0.990 & -2.435 & 0.978 & -2.564 & 0.994 & -0.724 & 0.988 & -2.059 & 0.995 & -0.503 & 0.983 & -1.979 & 1.000 & 1.594 \\
\hline 0.25 & 0.999 & -0.740 & 0.969 & -2.516 & 0.989 & -1.559 & 0.992 & -2.569 & 0.979 & -2.595 & 0.997 & -0.423 & 0.987 & -2.170 & 0.991 & -0.951 & 0.991 & -2.206 & 1.000 & 1.889 \\
\hline 0.30 & 1.000 & -0.447 & 0.974 & -2.390 & 0.985 & -1.916 & 0.993 & -2.582 & 0.979 & -2.355 & 0.997 & -0.474 & 0.991 & -1.846 & 0.991 & -1.304 & 0.994 & -2.153 & 0.998 & 1.613 \\
\hline 0.35 & 0.999 & -0.618 & 0.983 & -2.135 & 0.987 & -1.474 & 0.997 & -1.513 & 0.980 & -1.808 & 0.997 & -0.666 & 0.993 & -1.569 & 0.989 & -1.668 & 0.995 & -2.104 & 0.999 & 1.267 \\
\hline 0.40 & 0.999 & -1.241 & 0.985 & -2.011 & 0.986 & -1.703 & 0.997 & -1.540 & 0.980 & -2.209 & 0.997 & -1.205 & 0.994 & -1.852 & 0.990 & -1.970 & 0.995 & -1.770 & 0.999 & 0.845 \\
\hline 0.45 & 0.999 & -1.665 & 0.986 & -2.232 & 0.985 & -1.801 & 0.997 & -1.430 & 0.980 & -2.318 & 0.997 & -2.340 & 0.995 & -1.794 & 0.989 & -2.249 & 0.995 & -2.001 & 0.999 & 0.219 \\
\hline 0.50 & 0.999 & -1.754 & 0.986 & -2.259 & 0.985 & -1.575 & 0.997 & -1.134 & 0.980 & -2.374 & 0.997 & -2.593 & 0.995 & -1.918 & 0.989 & -2.380 & 0.995 & -2.006 & 0.998 & -0.707 \\
\hline 0.55 & 0.998 & -1.936 & 0.986 & -2.484 & 0.984 & -1.929 & 0.998 & -1.158 & 0.981 & -2.358 & 0.997 & -2.194 & 0.994 & -2.434 & 0.989 & -2.251 & 0.995 & -1.757 & 0.997 & -1.506 \\
\hline 0.60 & 0.998 & -1.463 & 0.984 & -2.482 & 0.983 & -1.604 & 0.998 & -1.050 & 0.981 & -1.135 & 0.997 & -1.277 & 0.989 & -2.001 & 0.988 & -1.974 & 0.995 & -2.071 & 0.996 & -1.561 \\
\hline 0.65 & 0.998 & -1.312 & 0.978 & -2.351 & 0.982 & -1.291 & 0.996 & -1.488 & 0.982 & -1.445 & 0.994 & -1.252 & 0.990 & -1.906 & 0.988 & -1.705 & 0.995 & -2.394 & 0.993 & -1.954 \\
\hline 0.70 & 0.998 & -2.199 & 0.974 & -2.393 & 0.979 & -1.914 & 0.997 & -1.068 & 0.982 & -1.507 & 0.994 & -0.954 & 0.988 & -1.957 & 0.988 & -1.887 & 0.995 & -2.199 & 0.992 & -1.025 \\
\hline 0.75 & 0.998 & -2.296 & 0.964 & -2.570 & 0.974 & -2.265 & 0.997 & -0.833 & 0.983 & -1.852 & 0.995 & -0.580 & 0.987 & -2.204 & 0.989 & -1.549 & 0.993 & -2.279 & 0.990 & -0.687 \\
\hline 0.80 & 0.998 & -2.023 & 0.945 & -1.409 & 0.960 & -1.981 & 1.001 & 0.404 & 0.983 & -1.669 & 0.994 & -0.719 & 0.990 & -1.955 & 0.988 & -1.159 & 0.984 & -2.333 & 0.986 & -0.458 \\
\hline 0.85 & 0.998 & -0.670 & 0.934 & -1.137 & 0.958 & -1.474 & 1.005 & 1.759 & 0.980 & -2.439 & 0.988 & -1.295 & 0.993 & -1.879 & 0.989 & -0.694 & 0.983 & -2.215 & 0.966 & -0.682 \\
\hline 0.90 & 0.999 & -0.115 & 0.924 & -0.807 & 0.979 & -0.475 & 1.004 & 0.154 & 0.973 & -1.973 & 0.977 & -0.773 & 0.984 & -0.803 & 0.996 & -0.137 & 0.982 & -0.974 & 0.888 & -1.976 \\
\hline \multirow[t]{2}{*}{0.95} & 0.991 & -0.690 & 0.864 & -1.057 & 81 & -0.280 & 1.000 & -0.010 & 0.969 & -0.668 & 0.972 & -0.439 & 0.980 & -0.551 & 0.950 & -0.398 & 0.984 & -0.674 & 0.760 & -1.957 \\
\hline & \multicolumn{4}{|c|}{ Oman } & \multicolumn{4}{|c|}{ Qatar } & \multicolumn{4}{|c|}{ Saudi Arabia } & \multicolumn{5}{|c|}{ Turkey } & \multicolumn{3}{|c|}{ UAE } \\
\hline 0.05 & 1.003 & 0.066 & 0.984 & -0.241 & 0.975 & -0.638 & 0.978 & -0.393 & 0.977 & -0.439 & 1.001 & 0.014 & 0.970 & -0.736 & 0.952 & -0.631 & 1.010 & 0.331 & 0.985 & -0.090 \\
\hline 0.10 & 1.002 & 0.215 & 0.985 & -1.460 & 0.994 & -0.740 & 0.990 & -1.390 & 0.976 & -1.174 & 1.018 & 0.354 & 0.975 & -1.000 & 0.957 & -1.454 & 1.009 & 0.746 & 0.993 & -0.410 \\
\hline 0.15 & 1.002 & 1.913 & 0.990 & -1.515 & 0.994 & -0.854 & 0.990 & -2.075 & 0.979 & -1.996 & 1.000 & -0.010 & 0.975 & -1.141 & 0.965 & -1.895 & 1.004 & 1.118 & 0.991 & -0.545 \\
\hline 0.20 & 1.002 & 0.915 & 0.993 & -1.025 & 0.995 & -0.972 & & -2.273 & 0.977 & -2.084 & 1.000 & & 0.976 & -1.200 & 0.962 & -1.842 & 0.999 & -0.224 & 0.992 & -0.659 \\
\hline 0.25 & 1.001 & 0.842 & 0.990 & -1.449 & 0.995 & -1.003 & 0.997 & -1.458 & 0.980 & -1.989 & 1.002 & 0.182 & 0.977 & -1.947 & 0.959 & -1.481 & 0.997 & -0.904 & 0.992 & -0.731 \\
\hline 0.30 & 1.001 & 0.608 & 0.993 & -1.206 & 0.995 & -1.211 & 0.997 & -1.510 & 0.983 & -1.709 & 1.002 & 0.290 & 0.977 & -1.814 & 0.967 & -1.659 & 0.996 & -1.679 & 0.994 & -1.063 \\
\hline 0.35 & 1.000 & 0.106 & 0.994 & -1.205 & 0.996 & -1.188 & 0.997 & -1.581 & 0.986 & -1.760 & 1.003 & 0.560 & 0.985 & -2.360 & 0.985 & -1.838 & 0.996 & -1.762 & 0.994 & -1.463 \\
\hline 0.40 & 1.000 & -0.199 & 0.995 & -1.846 & 0.997 & -1.583 & 0.997 & -1.785 & 0.989 & -1.938 & 0.997 & -0.632 & 0.993 & -1.986 & 0.989 & -2.011 & 0.996 & -2.115 & 0.993 & -1.707 \\
\hline 0.45 & 0.997 & -1.151 & 0.995 & -1.986 & 0.998 & -1.328 & 0.998 & -2.482 & 0.989 & -2.192 & 0.995 & -1.031 & 0.996 & -2.290 & 0.990 & -1.870 & 0.996 & -2.204 & 0.993 & -1.986 \\
\hline 0.50 & 0.997 & -1.418 & 0.995 & -2.321 & 0.999 & -0.400 & 0.998 & -2.421 & 0.987 & -2.094 & 0.995 & -0.936 & 0.996 & -1.624 & 0.991 & -2.434 & 0.995 & -1.890 & 0.993 & -1.540 \\
\hline 0.55 & 0.996 & -1.522 & 0.995 & -2.187 & 1.000 & -0.183 & 0.998 & -2.122 & 0.983 & -2.499 & 0.993 & -1.132 & 0.996 & -1.315 & 0.991 & -2.343 & 0.994 & -1.564 & 0.993 & -1.789 \\
\hline 0.60 & 0.996 & -2.485 & 0.995 & -2.153 & 1.001 & 0.245 & 0.998 & -2.079 & 0.981 & -2.467 & 0.990 & -2.053 & 0.996 & -1.638 & 0.993 & -2.247 & 0.994 & -1.977 & 0.993 & -2.192 \\
\hline 0.65 & 0.998 & -1.172 & 0.995 & -1.640 & 1.000 & -0.156 & 0.998 & -1.408 & 0.973 & -2.470 & 0.987 & -2.253 & 0.996 & -1.667 & 0.992 & -1.989 & 0.993 & -1.798 & 0.993 & -2.461 \\
\hline 0.70 & 0.998 & -0.590 & 0.994 & -1.172 & 0.998 & -0.557 & 0.998 & -1.285 & 0.969 & -2.103 & 0.986 & -2.437 & 0.989 & -1.639 & 0.987 & -2.507 & 0.993 & -1.569 & 0.992 & -1.392 \\
\hline 0.75 & 0.995 & -0.977 & 0.993 & -1.040 & 0.998 & -0.474 & 0.998 & -0.552 & 0.970 & -2.349 & 0.977 & -2.140 & 0.988 & -1.644 & 0.986 & -2.490 & 0.992 & -1.599 & 0.991 & -1.123 \\
\hline 0.80 & 0.991 & -1.027 & 1.001 & 0.136 & 0.994 & -1.456 & 0.998 & -0.270 & 0.970 & -1.941 & 0.975 & -2.211 & 0.987 & -1.705 & 0.984 & -2.266 & 0.986 & -2.143 & 0.987 & -1.108 \\
\hline 0.85 & 0.975 & -2.363 & 1.002 & 0.192 & 0.995 & -1.109 & 1.001 & 0.096 & 0.971 & -1.702 & 0.978 & -2.004 & 0.991 & -2.249 & 0.973 & -2.066 & 0.982 & -2.099 & 0.984 & -1.052 \\
\hline 0.90 & 0.960 & -1.002 & 0.990 & -0.503 & 0.999 & -0.064 & 0.986 & -0.939 & 0.971 & -1.961 & 0.974 & -0.370 & 0.993 & -0.364 & 0.965 & -0.788 & 0.984 & -0.671 & 0.970 & -1.083 \\
\hline 0.95 & 0.934 & -1.242 & 0.972 & -0.294 & 0.999 & -0.023 & 0.996 & -0.187 & 0.966 & -1.010 & 0.974 & -0.236 & 0.994 & -0.159 & 0.945 & -0.826 & 0.976 & -0.819 & 0.934 & -0.597 \\
\hline
\end{tabular}

Source: Authors Estimation

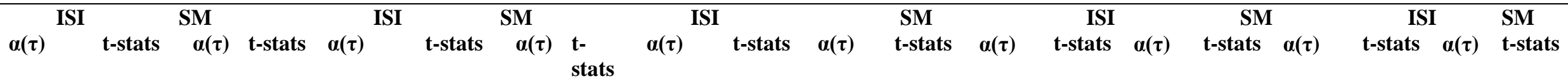




\begin{tabular}{|c|c|c|c|c|c|c|c|c|c|c|c|c|c|c|c|c|c|c|c|c|}
\hline 05 & .001 & 058 & 971 & -0.128 & 922 & .559 & 988 & .292 & 972 & -0.583 & 992 & 0.114 & 980 & -0.369 & 978 & -0.250 & 973 & -1.748 & .001 & 674 \\
\hline 0.10 & 1.001 & 0.174 & .979 & -0.166 & 1.010 & 0.379 & .989 & -0.354 & .979 & -2.027 & .001 & 0.031 & 0.985 & -1.619 & 0.984 & -0.824 & 0.986 & -2.446 & 1.000 & 0.904 \\
\hline 0.15 & 0.998 & -1.298 & .972 & -0.865 & .000 & 0.012 & .988 & -2.252 & .980 & -2.383 & .996 & -0.375 & .990 & -1.950 & .995 & 0.362 & 0.986 & -2.044 & .000 & 1.274 \\
\hline 20 & 0.998 & .343 & 968 & -2.582 & .991 & 1.007 & .990 & -2.435 & 78 & 2.564 & 94 & -0.724 & 88 & -2.059 & 995 & 503 & & -1.979 & & 1.594 \\
\hline 0.25 & 0.999 & -0.740 & 969 & -2.516 & .989 & 1.559 & .992 & -2.569 & .979 & -2.595 & .997 & & .987 & -2.170 & .991 & 0.951 & 991 & -2.206 & & 1.889 \\
\hline 0.30 & 1.000 & -0.447 & .974 & -2.390 & .985 & -1.916 & 93 & -2.582 & 979 & -2.355 & 97 & -0.474 & 991 & -1.846 & 91 & 1.304 & 94 & -2.153 & & 1.613 \\
\hline 35 & 0.999 & -0.618 & .983 & -2.135 & .987 & 1.474 & .997 & -1.513 & .980 & 1.808 & .997 & -0.666 & 993 & -1.569 & .989 & 1.668 & 995 & & & 1.267 \\
\hline 40 & 0.999 & -1.241 & .985 & -2.011 & .986 & -1.703 & 997 & -1.540 & & -2.209 & .997 & -1.205 & 994 & & .990 & -1.970 & & -1.770 & & 0.845 \\
\hline 45 & 0.999 & -1.665 & 986 & -2.232 & 985 & 1.801 & 97 & 1.430 & & -2.318 & & & 95 & & & & & & & 0.219 \\
\hline 50 & 0.999 & -1.754 & .986 & -2.259 & .985 & -1.575 & .997 & -1.134 & & -2.374 & 97 & -2.593 & 995 & -1.918 & & -2.380 & & -2.006 & & -0.707 \\
\hline & 98 & -1.936 & 6 & -2.484 & & -1.929 & 98 & 1.158 & & -2.358 & & -2.194 & & & & & & & & -1.506 \\
\hline 60 & 0.998 & -1.463 & 4 & -2.482 & & & & -1.050 & & & & -1.277 & 89 & -2 . & 88 & 74 & 95 & & 96 & -1.561 \\
\hline 65 & 0.998 & -1.312 & 8 & -2.351 & 32 & -1.291 & 96 & -1.488 & 32 & 45 & 94 & -1.252 & & & & & & -2 & & 954 \\
\hline 70 & 0.998 & & 0.9 & -2.393 & & & & & & & & & & & & & & & & -1.025 \\
\hline 75 & 0.998 & -2.296 & 0.964 & -2.570 & 0.974 & -2.265 & 97 & -0.833 & 83 & -1.852 & 95 & -0.580 & 87 & -2.204 & 89 & 49 & 93 & -2.279 & 990 & -0.687 \\
\hline 80 & 0.998 & -2.023 & & -1.409 & & & & 0.404 & & & & & & & & & & & & 58 \\
\hline 85 & 0.998 & -0.670 & .934 & -1.137 & 0.958 & 1.474 & 05 & 1.759 & & -2.439 & & -1.295 & 93 & -1 . & & 94 & 83 & -2.215 & 66 & -0.682 \\
\hline 90 & 0.999 & -0.115 & 4 & -0.807 & & -0.475 & & & & & & & & & & & & & & -1.976 \\
\hline & & & & & & & & -0.010 & & & & & & & & & & & & 57 \\
\hline \multicolumn{3}{|c|}{ Oman } & \multicolumn{4}{|c|}{ Qatar } & & & \multicolumn{4}{|c|}{ Saudi Arabia } & \multicolumn{5}{|c|}{ Turkey } & \multicolumn{3}{|c|}{ UA } \\
\hline 0.05 & & & & & & & & & & & & & & & & & & & & \\
\hline 10 & 1.002 & 0.215 & 0.985 & 1.460 & .994 & -0.740 & .990 & -1.390 & 76 & -1.174 & 18 & & 75 & -1 . & & & 09 & 0.746 & 93 & -0.410 \\
\hline 15 & 1.002 & 1.913 & 0.990 & -1.515 & 0.994 & -0.854 & .990 & -2.075 & 0.979 & -1.996 & 1.000 & 10 & & & & & & 18 & 91 & -0.545 \\
\hline 20 & 1.002 & 0.915 & 3 & 1.025 & 95 & -0.972 & 92 & -2.273 & & -2.084 & 1.000 & & & -1 . & & & & -0.224 & & -0.659 \\
\hline 25 & 1.001 & 0.842 & 0.9 & 1.449 & 5 & -1.003 & 0.997 & -1.458 & & -1.989 & 1.002 & & & & & & & -0.904 & & -0.731 \\
\hline 30 & 1.001 & 0.608 & 0.9 & 1.206 & & -1.211 & & -1.510 & & -1.709 & & & & & & & & -1. & & -1.063 \\
\hline 35 & 1.000 & 0.106 & 4 & -1.205 & & -1.188 & .997 & -1.581 & & -1.760 & & & & & & & & & & -1.463 \\
\hline 40 & & & & & & & & & & & & & & & & & & & & \\
\hline 45 & 0.997 & -1.151 & 0.995 & -1.986 & & -1.328 & & -2.482 & & -2.192 & & 1 & & -2.290 & & & & -2.2 & & -1.986 \\
\hline 0.50 & 0.997 & & & -2.321 & & & & & & & & & & & & & & & & -1.540 \\
\hline 55 & 0.996 & -1.522 & 0.995 & -2.187 & & -0.183 & & -2.122 & & -2.499 & & & & & & & & -1.564 & & -1.789 \\
\hline 0.60 & 0.996 & -2.485 & 0.995 & -2.153 & 1.001 & 0.245 & 0.998 & -2.079 & 81 & -2.467 & 0.990 & -2.053 & 96 & -1.638 & 93 & -2.247 & 94 & -1.977 & 93 & -2.192 \\
\hline 0.65 & 0.998 & -1.172 & 0.995 & -1.640 & & -0.156 & & -1.408 & & -2.470 & & & & & & & & & & -2.461 \\
\hline 0.70 & 0.998 & -0.590 & 0.994 & -1.172 & 0.998 & -0.557 & 0.998 & -1.285 & & -2.103 & 0.986 & -2.437 & 0.989 & -1.639 & & -2.507 & & -1.569 & 92 & -1.392 \\
\hline 0.75 & 0.995 & -0.977 & 0.993 & -1.040 & & & & -0.552 & & -2.349 & 0.977 & & & & & & & -1.5 & & -1.123 \\
\hline 0.80 & 0.991 & -1.027 & 1.001 & 0.136 & 0.994 & -1.456 & 0.998 & -0.270 & & -1.941 & 0.975 & -2.211 & 0.987 & -1.705 & & -2.266 & & -2.143 & 0.987 & -1.108 \\
\hline 0.85 & 0.975 & -2.363 & 1.002 & 0.192 & 0.995 & -1.109 & 1.001 & 0.096 & 0.971 & -1.702 & & -2.004 & & & & -2.066 & & -2.099 & & -1.052 \\
\hline 90 & 0.960 & -1.002 & .990 & -0.503 & & & 86 & -0.939 & & -1.961 & 0.974 & -0.370 & & & & -0.788 & & -0.671 & 0.970 & -1.083 \\
\hline 0.95 & 0.934 & -1.242 & 0.972 & -0.294 & 0.999 & -0.023 & 0.996 & -0.187 & 0.966 & -1.010 & 0.974 & -0.236 & 0.994 & -0.159 & 0.945 & -0.826 & 0.976 & -0.819 & 0.934 & -0.597 \\
\hline
\end{tabular}

Table1: Quantile Unit Root Test

Table 2(a) and 2(b) employ the Xiao (2009) cointegration test to confirm that long-run association between the variables by applying the grid of 10 quantiles (0.01-0.95) in logarithm form. The critical values are produced by using 1000 Monte Carlo simulations. CV1, CV5, and CV10 are the 
critical values at $1 \%, 5 \%$ and $10 \%$ level of significance, respectively. Supremum norm values of coefficients $(\beta$ and $\Gamma)$ are larger than all the critical values showing that the cointegration association between the variables changes over quantile distribution which is the sign of a significant nonlinear or asymmetric long-run association. 
Table2(a): $\quad$ Quantile Cointegration Test Results (Conventional Bond Market and Islamic Stock Index)

$\begin{array}{lccccc}\text { Country } & \text { Coefficient } & \text { Sup }_{\boldsymbol{\tau}}|\mathbf{V} \mathbf{n}(\boldsymbol{\tau})| & \mathbf{C V 1} & \mathbf{C V 5} & \mathbf{C V 1 0} \\ \text { Bahrain } & \beta & 68568.30 & 58359.38 & 57206.27 & 54777.74 \\ \text { CBMt vs. ISIt } & \Gamma & 2466.69 & 1489.24 & 1458.50 & 1480.22 \\ \text { Brunei } & \beta & 8336.25 & 5293.26 & 3144.06 & 2547.33 \\ \text { CBMt vs. ISIt } & \Gamma & 166.67 & 104.35 & 49.62 & 39.31 \\ \text { Kuwait } & \beta & 3948.92 & 3788.25 & 2452.83 & 2054.07 \\ \text { CBMt vs. ISIt } & \Gamma & 162.729 & 150.800 & 46.076 & 49.765 \\ \text { Libya } & \beta & 8646.53 & 2933.04 & 2413.40 & 1420.20 \\ \text { CBMt vs. ISIt } & \Gamma & 166.70 & 79.97 & 60.80 & 50.72 \\ \quad \text { Malaysia } & \beta & 39726.06 & 27489.40 & 13665.41 & 5634.60 \\ \text { CBMt vs. ISIt } & \Gamma & 1299.47 & 1009.52 & 797.40 & 569.59 \\ \text { Oman } & \beta & 81833.54 & 67123.30 & 37922.33 & 21848.22 \\ \text { CBMt vs. ISIt } & \Gamma & 993.67 & 646.61 & 588.50 & 564.42 \\ \text { Qatar } & \beta & 19777.30 & 15989.22 & 11169.07 & 7647.05 \\ \text { CBMt vs. ISIt } & \Gamma & 657.55 & 554.00 & 532.10 & 224.68 \\ \text { Saudi Arabia } & \beta & 9382.00 & 7200.00 & 5900.70 & 2622.00 \\ \text { CBMt vs. ISIt } & \Gamma & 249.67 & 161.40 & 129.58 & 99.09 \\ \text { Turkey } & \beta & 8754.51 & 6730.00 & 4770.11 & 1488.90 \\ \text { CBMt vs. ISIt } & \Gamma & 399.10 & 200.60 & 100.01 & 97.00 \\ \text { UAE } & \beta & 7111.31 & 3492.10 & 3088.11 & 2225.30 \\ \text { CBMt vs. ISIt } & \Gamma & 600.40 & 300.84 & 215.17 & 117.11\end{array}$

Note: This table presents the results of the quantile cointegration test of Xiao (2009) for the conventional bonds markets (CBM) and ISI (Islamic stock index). We test the stability of the coefficients $\beta$ and $\Gamma$ in the quantile cointegration model.

Table2(b): Quantile Cointegration Test Results (Sukuk Market and Islamic Stock Index)

$\begin{array}{lccccc}\text { Country } & \text { Coefficient } & \mathbf{S u p}_{\boldsymbol{\tau}}\left|\mathbf{V}_{\mathbf{n}}(\boldsymbol{\tau})\right| & \mathbf{C V 1} & \mathbf{C V 5} & \mathbf{C V 1 0} \\ \text { Bahrain } & \beta & 66773.33 & 60779.36 & 49405.89 & 29890.92 \\ \text { SMt vs. ISI } & \Gamma & 6525.92 & 1499.40 & 1066.50 & 940.42 \\ \text { Brunei } & \beta & 8001.20 & 5009.52 & 4033.00 & 3059.31 \\ \text { SMt vs. ISIt } & \Gamma & 179.80 & 109.63 & 66.81 & 51.91 \\ \text { Kuwait } & \beta & 4088.99 & 3077.28 & 2224.08 & 1155.00 \\ \text { SMt vs. ISI } & \Gamma & 133.72 & 120.66 & 78.00 & 61.76 \\ \text { Libya } & \beta & 6066.32 & 4022.00 & 3921.00 & 2255.22 \\ \text { SMt vs. ISIt } & \Gamma & 177.73 & 83.197 & 61.86 & 50.22 \\ \quad \text { Malaysia } & \beta & 51227.00 & 40755.00 & 30609.01 & 24097.70 \\ \text { SMt vs. ISIt } & \Gamma & 1099.88 & 1004.25 & 991.64 & 769.29 \\ \text { Oman } & \beta & 43819.90 & 22391.28 & 13981.44 & 9899.23 \\ \text { SMt vs. ISI } & \Gamma & 999.72 & 466.21 & 285.30 & 145.72 \\ \text { Qatar } & \beta & 66719.30 & 56797.32 & 40197.00 & 20679.00 \\ \text { SMt vs. ISIt } & \Gamma & 7646.55 & 5244.00 & 3401.11 & 2206.80 \\ \text { Saudi Arabia } & \beta & 2899.20 & 2088.34 & 1888.97 & 1635.55 \\ \text { SMt vs. ISI } & \Gamma & 261.72 & 155.44 & 134.80 & 111.01 \\ \text { Turkey } & \beta & 3366.61 & 2650.01 & 2138.19 & 1288.70 \\ \text { SMt vs. ISIt } & \Gamma & 428.90 & 311.80 & 181.70 & 111.20 \\ \text { UAE } & \beta & 6672.87 & 3991.24 & 2972.95 & 1326.75 \\ \text { SMt vs. ISIt } & \Gamma & 736.49 & 448.38 & 346.00 & 212.11\end{array}$

Note: This table presents the results of the quantile cointegration test of Xiao (2009) for the sukuk markets (SM) and ISI (Islamic stock index). We test the stability of the coefficients $\beta$ and 
$\Gamma$ in the quantile cointegration model.

\section{Quantile-on-Quantile Estimation}

This section describes the empirical results of the Quantile and Quantile analysis between conventional bond market, Sukuk market and Islamic stock index of the Top 10 Islamic stock markets.

Figure 1: Impact of conventional bond on Islamic Stocks

Quantile-on-Quantile (QQ) estimates of the slope coefficient, $\boldsymbol{\beta}_{\mathbf{1}}(\boldsymbol{\theta}, \boldsymbol{\tau})$

i).Bahrain

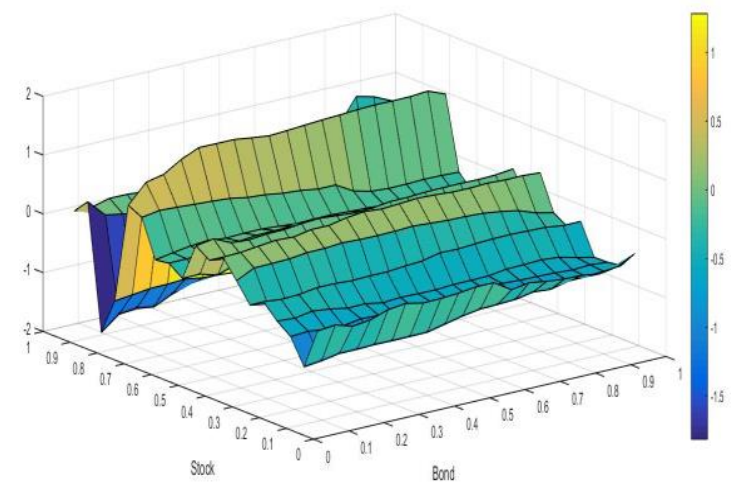

iii).Kuwait

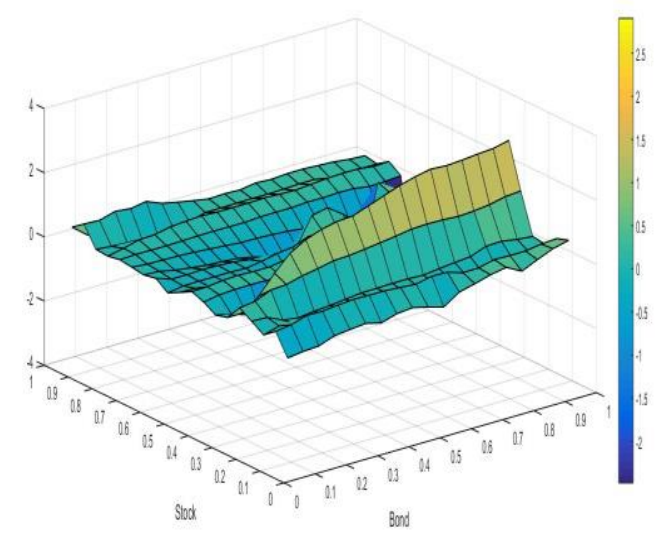

v).Malaysia

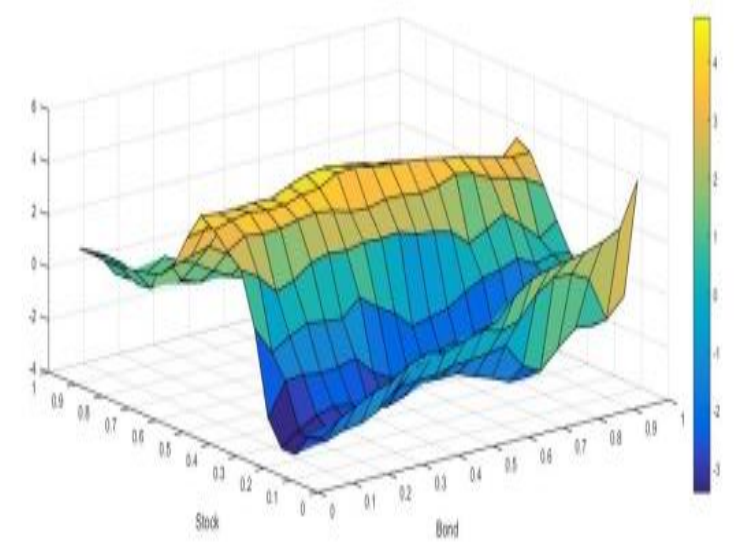

vii).Qatar ii). Brunei

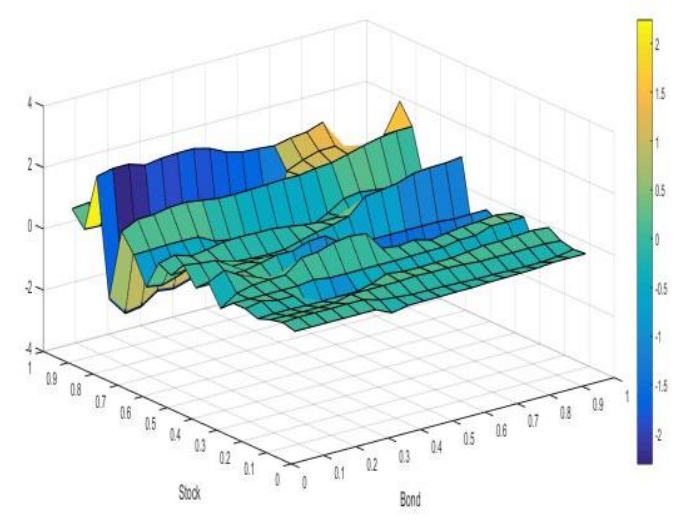

iv).Libya

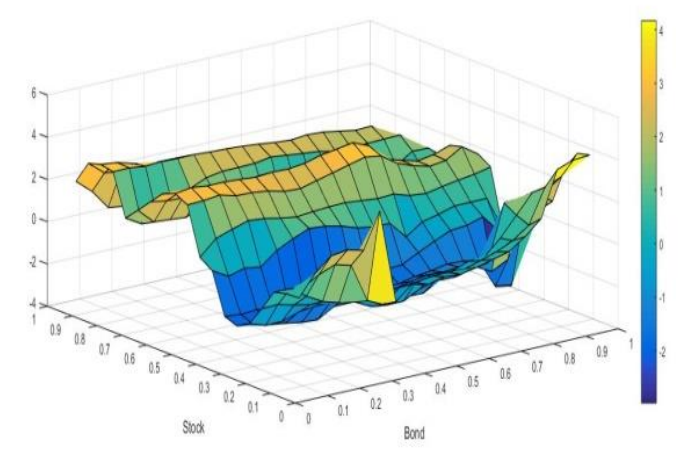

vi). Oman

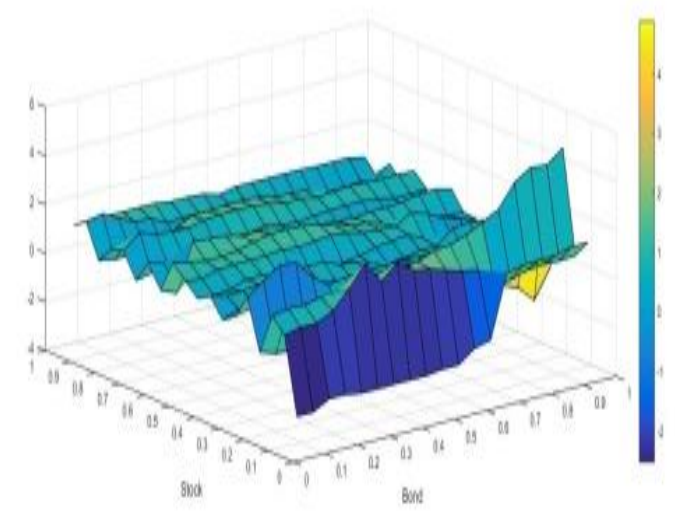

viii).Saudi Arabia 


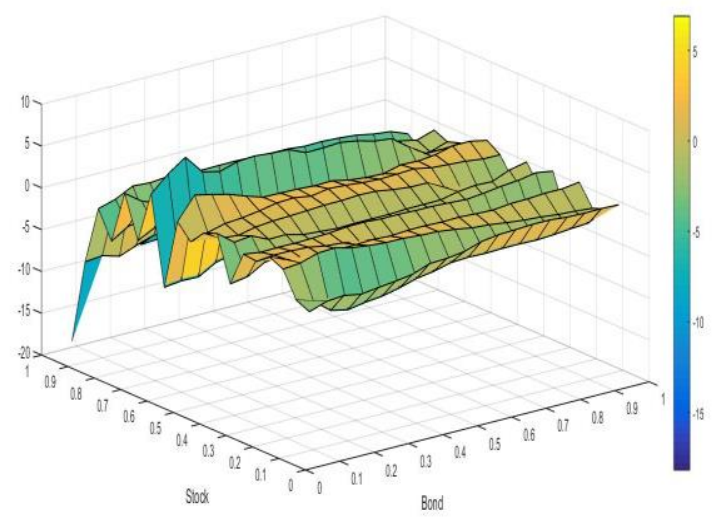

ix).Turkey

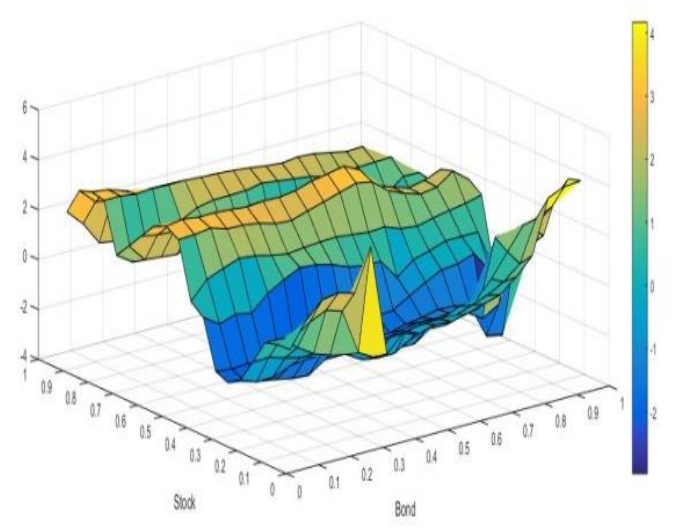

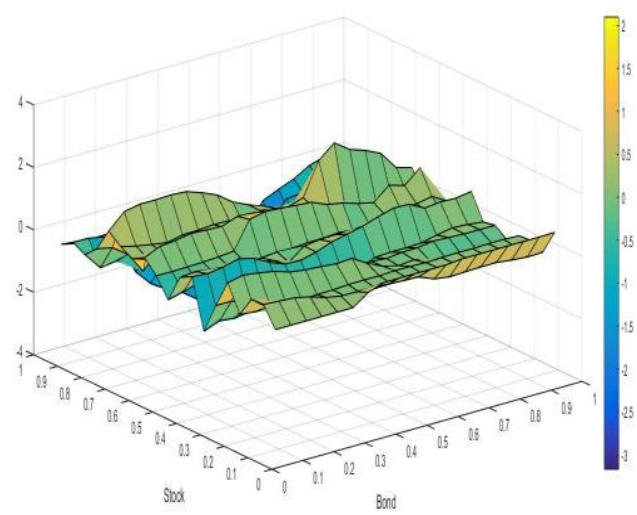

x).United Arab Emirates

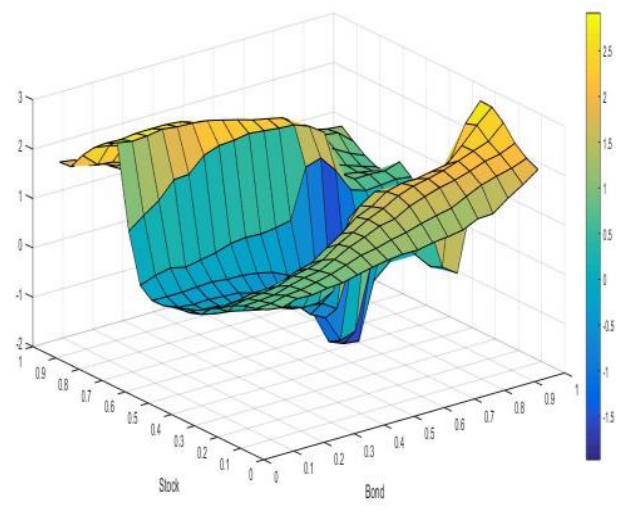

Note:Slope coefficient $\boldsymbol{\beta}_{1}(\boldsymbol{\theta}, \boldsymbol{\tau})$ are shown on the $\mathrm{z}$-axis against the quantiles of conventional bond on the $\mathrm{x}$-axis and the quantiles of Islamic stock on the $\mathrm{y}$-axis.

Figure 1 shows the impact of conventional bond on Islamic Stocks in top 10 Islamic stock markets. In Libya and Malaysia, the effect of conventional bond on Islamic stock is negative in the area which combines all the quantiles of conventional bond with lower to lower-middle quantiles of Islamic stock (0.05-0.40). This finding indicates that demand of conventional bond decreases in bearish market condition or when the market is in stress. The effect of conventional bond on Islamic stock is positive between the regions which combines the quantiles of conventional bond with upper-middle quantiles of Islamic stock (0.60-0.70) under bullish market conditions. For the Oman and Kuwait, a strong negative association is present in the area which combines all the quantiles of conventional bond with the lower quantiles of Islamic stock (0.10-0.30) under the bearish market condition.

Qatar and Saudi Arabia have a positive association between the conventional bond and Islamic stock. This effect is strong in the region which combines all the quantiles of conventional bond with lower-middle to upper-middle quantiles of Islamic stock (0.45-0.70). In Bahrain and Brunei, the overall effect of conventional bond on Islamic stock is also positive. A strong positive effect of conventional bond on Islamic stock is found at the upper-middle quantiles (0.60-0.75) of Islamic stock. A minor positive effect is also found at the lower quantile of both conventional bond and the Islamic stock under bearish market condition. However, a strong negative effect of conventional bond on Islamic stock is found in the area that combines all the quantiles of conventional bond with the upper quantiles of Islamic stock $(0.80-0.80)$ under bullish market conditions. This finding shows that demand of conventional bond decreases under bullish market condition or when the market is in stress.

In Turkey and the United Arab Emirates, the effect of conventional bond on Islamic stock is mixed. A strong negative association between the conventional bond and Islamic stock is found in the region which combines all the quantiles 
of conventional bond with lower to lower-middle quantiles of Islamic stock (0.05-0.40) in Turkey and lower-middle to upper-middle quantiles of Islamic stock in the United Arab Emirates which indicates that the demand of conventional bond decreases under the bearish market condition in Turkey. The effect of conventional bond on Islamic stock becomes strong and positive between the area which combines all the quantiles of conventional bond with lower to lower-middle and upper-middle to higher quantiles $(0.05-0.40 \& 0.70-0.95)$ of Islamic stock in the United Arab Emirates which shows that the demand of conventional bond increases under both bearish and bullish market conditions in United Arab Emirates.

The findings of the study confirmed that generally, at the high quantiles (bullish condition) of both Islamic stock markets and conventional bonds, a strong positive relationship exists in all the Islamic stock markets. While on the low quantiles (bearish condition) of both Islamic stock markets and conventional bonds, a mild negative relationship exists in all the Islamic stock markets. The findings of the study are aligning with the findings of Nasir and Farooq (2017) and Ahmed and Elsayed (2019). Hence the findings show an asymmetric relationship between these two variables.

Figure 2: Impact of Sakuk Market on Islamic Stocks

Quantile-on-Quantile (QQ) estimates of the slope coefficient, $\boldsymbol{\beta} \mathbf{1}(\boldsymbol{\theta}, \boldsymbol{\tau})$

\section{i).Bahrain}

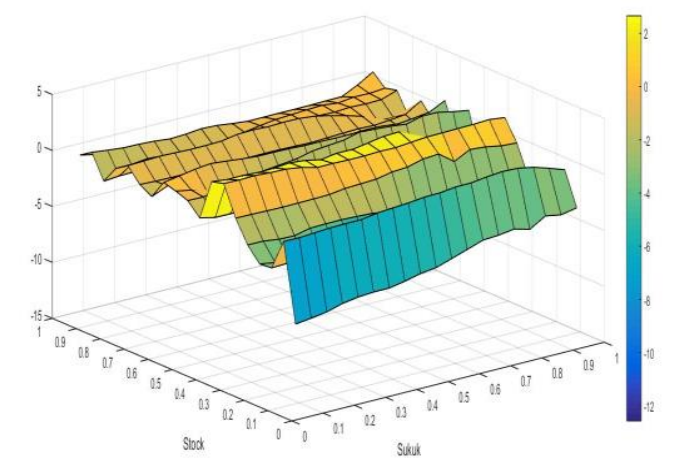

iii). Kuwait

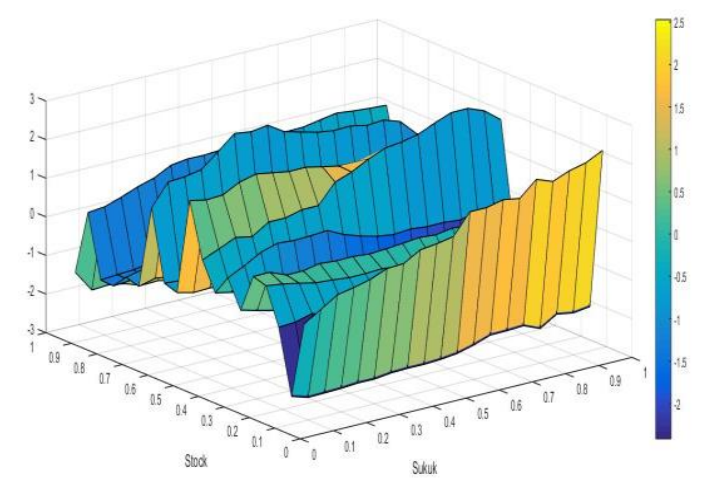

v).Malaysia ii). Brunei

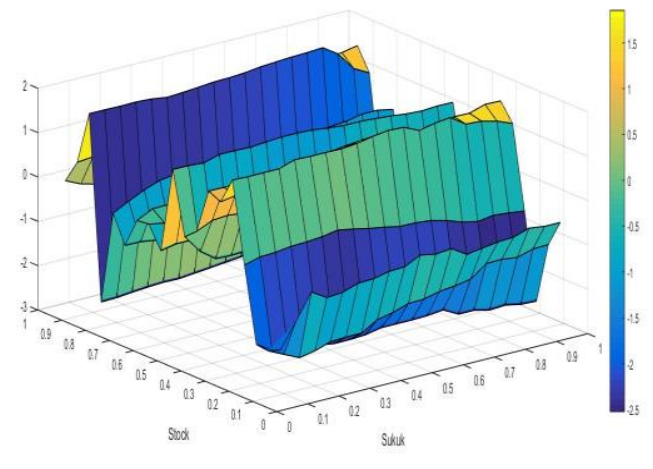

iv).Libya

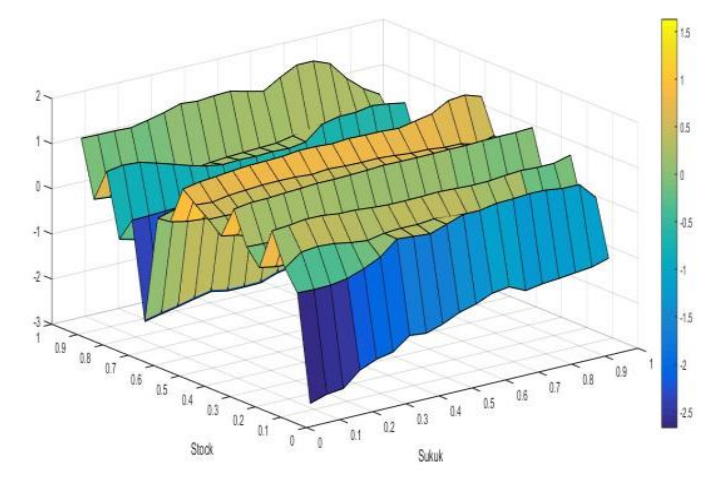

vi).Oman 


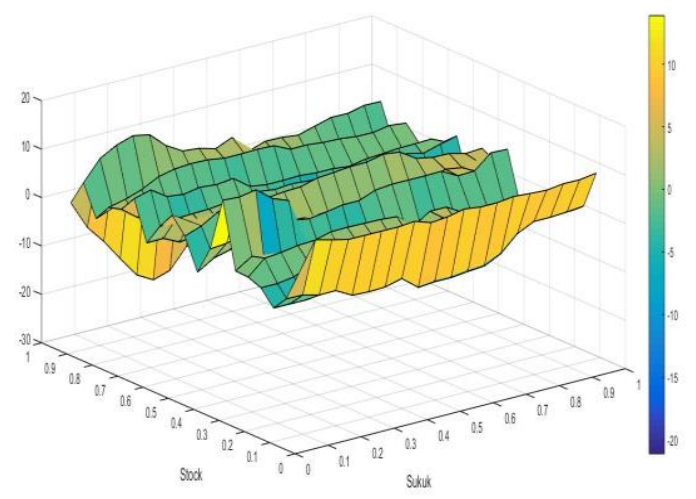

vii).Qatar

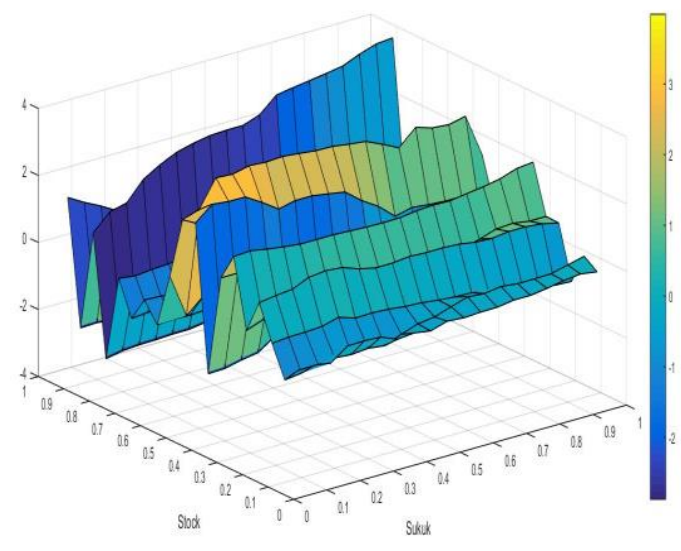

ix).Turkey

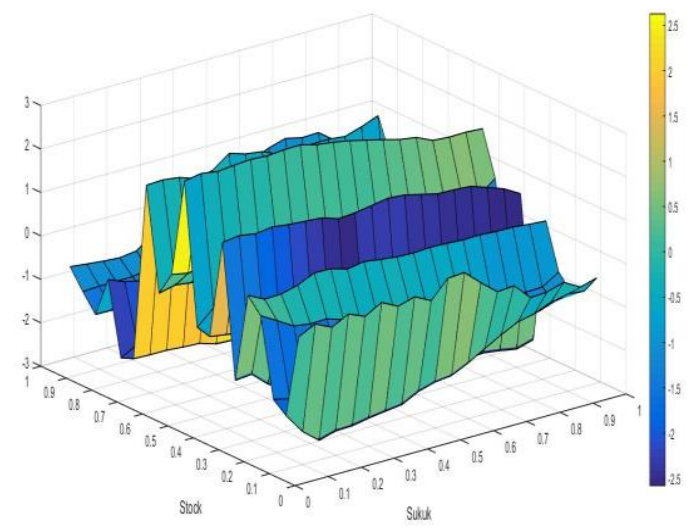

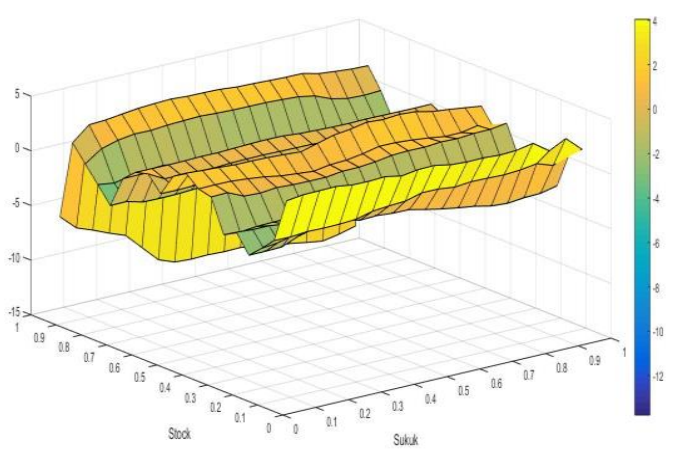

viii).Saudi Arabia

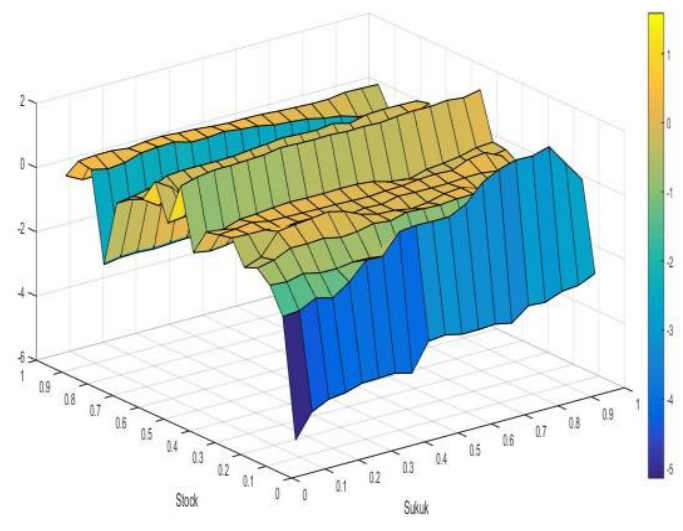

x).United Arab Emirates

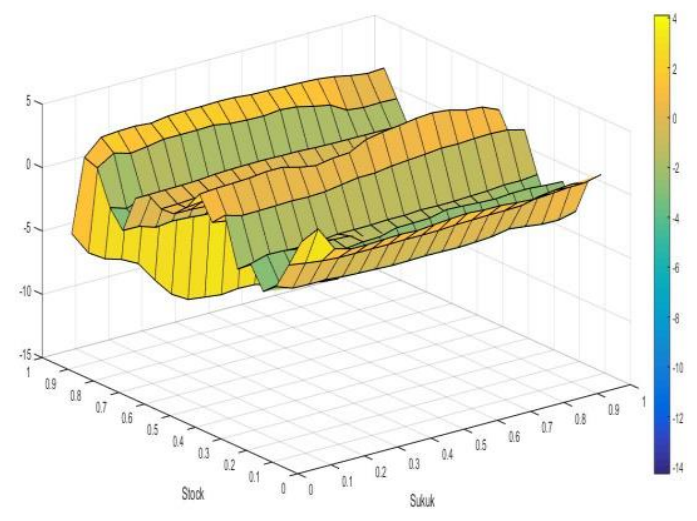

shown on the z-axis against

the quantiles of sukuk market on the $\mathrm{x}$-axis and the quantiles of Islamic stock on the $\mathrm{y}$-axis.

Figure 1 shows the impact of sukuk on Islamic stocks in top 10 Islamic stock markets. In Brunei and Qatar, the effect of sukuk market on Islamic stock is negative for the majority of the quantiles of both variables. This negative effect is very strong at lower and higher quantiles of sukuk market (0.05-0.20 \& 0.80-0.95) under bullish and bearish market conditions. In Kuwait and Turkey, the negative association between sukuk and Islamic stock becomes strong in the region which joins the quantiles of sukuk with higher quantiles of Islamic stock which demonstrates that demand of sukuk decreases under bullish market conditions. However, a positive impact of sukuk on Islamic stock is also 
observed in the area which connects the quantiles of sukuk with upper-middle quantiles of Islamic stock (0.65-0.80) in Turkey and lower quantiles of Islamic stock (0.05-0.30) in Kuwait under bullish and bearish market conditions, respectively.

In Malaysia, the effect of sukuk market on Islamic stock Index is weak and negative in lower-middle to upper-middle quantiles of Islamic stock (0.30-0.75). However, this effect becomes positive at the lower and upper quantiles of Islamic stocks. This finding clarifies that demand for sukuk increases in both bullish and bearish market conditions in Malaysia. For Oman and the United Arab Emirates, the effect of the sukuk market on Islamic stock is positive at the significant number of quantiles of both variables. This effect becomes strong in the area which connects the quantiles of sukuk with lower and upper quantiles of Islamic stocks in Oman (0.05-0.20\& 0.80-0.95) which means the demand of sukuk significantly increases under both bearish and bullish market conditions. On the other hand, the negative effect of sukuk becomes strong at the upper quantiles of Islamic stock in the United Arab Emirates, which shows the negative association between sukuk and Islamic stock in bullish market conditions.

For Bahrain, Libya and Saudi Arabia, the positive effect of sukuk on Islamic stock is dominant. The impact of sukuk on Islamic stock is positive in the area which merges all the quantiles of sukuk and lower-middle to upper-middle quantiles of Islamic stock (0.30-0.75) in Saudi Arabia and Libya while lower-middle to higher quantiles (0.30-0.95) of Islamic stock in Bahrain. However, the effect becomes negative in the region which connects the quantiles of sukuk with lower and higher quantiles of Islamic stock (0.05-0.30 \& 0.85-0.90) in Saudi Arabia and Libya, and lower quantiles of Islamic stock in Bahrain. The findings indicate that the demand for sukuk decreases under both bullish and bearish market conditions in Saudi Arabia and Libya while only bearish market condition in Bahrain.

The findings of the study confirmed that generally, at the low quantiles (bearish condition) of both Islamic stock markets and Islamic sukuk market, a strong negative relationship exists in all the Islamic stock markets. While on the high quantiles (bullish condition) of both Islamic stock markets and Islamic sukuk market, a mild positive relationship exists in all the Islamic stock markets. The findings of the study are aligning with the study of Naifar (2016). Hence the findings show an asymmetric relationship between these two variables. 
Table 3(a): Quantile Granger Causality (Conventional Bond Market and Islamic Stock Index)

\begin{tabular}{|c|c|c|c|c|c|c|c|c|c|c|c|c|c|c|c|c|c|c|c|}
\hline \multicolumn{20}{|c|}{ Bahrain } \\
\hline Bahrain & 0.05 & 0.10 & 0.15 & 0.20 & 0.25 & 0.30 & 0.35 & 0.40 & 0.45 & 0.50 & 0.55 & 0.60 & 0.65 & 0.70 & 0.75 & $\mathbf{0 . 8 0}$ & 0.85 & 0.90 & 0.95 \\
\hline$\Delta \mathrm{ISI}_{\mathrm{t}}$ to $\Delta \mathrm{CBM}_{\mathrm{t}}$ & 0.000 & 0.000 & 0.000 & 0.000 & 0.000 & 0.000 & 0.000 & 0.000 & 0.000 & 0.120 & 0.000 & 0.000 & 0.000 & 0.000 & 0.000 & 0.000 & 0.000 & 0.000 & 0.000 \\
\hline$\Delta \mathrm{CBM}_{\mathrm{t}}$ to $\Delta \mathrm{ISI}_{\mathrm{t}}$ & 0.000 & 0.000 & 0.000 & 0.000 & 0.000 & 0.000 & 0.000 & 0.000 & 0.000 & 0.759 & 0.000 & 0.000 & 0.000 & 0.000 & 0.000 & 0.000 & 0.000 & 0.000 & 0.000 \\
\hline \multicolumn{20}{|c|}{ Brunei } \\
\hline Brunei & 0.050 & 0.10 & 0.15 & 0.20 & 0.250 & 0.30 & 0.35 & 0.40 & 0.45 & 0.500 & 0.55 & 0.60 & 0.65 & 0.70 & 0.75 & 0.80 & 0.85 & 0.90 & 0.950 \\
\hline$\Delta \mathrm{ISI}_{\mathrm{t}}$ to $\Delta \mathrm{CBM}_{\mathrm{t}}$ & 0.528 & 0.000 & 0.000 & 0.000 & 0.000 & 0.000 & 0.000 & 0.000 & 0.000 & 0.009 & 0.000 & 0.000 & 0.000 & 0.000 & 0.000 & 0.000 & 0.000 & 0.000 & 0.000 \\
\hline$\Delta \mathrm{CBM}_{\mathrm{t}}$ to $\Delta \mathrm{ISI}_{\mathrm{t}}$ & 0.000 & 0.000 & 0.000 & 0.000 & 0.000 & 0.000 & 0.000 & 0.000 & 0.000 & 0.667 & 0.000 & 0.000 & 0.000 & 0.000 & 0.000 & 0.000 & 0.000 & 0.000 & 0.000 \\
\hline \multicolumn{20}{|c|}{ Kuwait } \\
\hline Kuwait & 0.050 & 0.10 & 0.15 & 0.20 & 0.250 & 0.30 & 0.35 & 0.40 & 0.45 & 0.500 & 0.55 & 0.60 & 0.65 & 0.70 & 0.75 & 0.80 & 0.85 & 0.90 & 0.950 \\
\hline$\Delta \mathrm{ISI}_{\mathrm{t}}$ to $\Delta \mathrm{CBM}_{\mathrm{t}}$ & 0.000 & 0.000 & 0.000 & 0.000 & 0.000 & 0.000 & 0.000 & 0.000 & 0.000 & 0.046 & 0.000 & 0.000 & 0.000 & 0.000 & 0.000 & 0.000 & 0.000 & 0.000 & 0.000 \\
\hline$\Delta \mathrm{CBM}_{\mathrm{t}}$ to $\Delta \mathrm{ISI}_{\mathrm{t}}$ & 0.000 & 0.000 & 0.000 & 0.000 & 0.000 & 0.000 & 0.000 & 0.000 & 0.000 & 0.931 & 0.000 & 0.000 & 0.000 & 0.000 & 0.000 & 0.000 & 0.000 & 0.000 & 0.000 \\
\hline Libya & 0.050 & 0.10 & 0.15 & 0.20 & 0.250 & 0.30 & 0.35 & 0.40 & 0.45 & 0.500 & 0.55 & 0.60 & 0.65 & 0.70 & 0.75 & 0.80 & 0.85 & 0.90 & 0.950 \\
\hline$\Delta \mathrm{ISI}_{\mathrm{t}}$ to $\Delta \mathrm{CBM}_{\mathrm{t}}$ & 0.000 & 0.000 & 0.000 & 0.000 & 0.000 & 0.000 & 0.000 & 0.000 & 0.000 & 0.829 & 0.000 & 0.000 & 0.000 & 0.000 & 0.000 & 0.000 & 0.000 & 0.000 & 0.000 \\
\hline$\Delta \mathrm{CBM}_{\mathrm{t}}$ to $\Delta \mathrm{ISI}_{\mathrm{t}}$ & 0.000 & 0.000 & 0.000 & 0.000 & 0.000 & 0.000 & 0.000 & 0.000 & 0.000 & 0.639 & 0.000 & 0.000 & 0.000 & 0.000 & 0.000 & 0.000 & 0.000 & 0.000 & 0.000 \\
\hline \multicolumn{20}{|c|}{ Malaysia } \\
\hline Malaysia & 0.050 & 0.10 & 0.15 & 0.20 & 0.250 & 0.30 & 0.35 & 0.40 & 0.45 & 0.500 & 0.55 & 0.60 & 0.65 & 0.70 & 0.75 & 0.80 & 0.85 & 0.90 & 0.950 \\
\hline$\Delta \mathrm{ISI}_{\mathrm{t}}$ to $\Delta \mathrm{CBM}_{\mathrm{t}}$ & 0.000 & 0.000 & 0.000 & 0.000 & 0.000 & 0.000 & 0.000 & 0.000 & 0.000 & 0.255 & 0.000 & 0.000 & 0.000 & 0.000 & 0.000 & 0.000 & 0.000 & 0.000 & 0.000 \\
\hline$\Delta \mathrm{CBM}_{\mathrm{t}}$ to $\Delta \mathrm{ISI}_{\mathrm{t}}$ & 0.000 & 0.000 & 0.000 & 0.000 & 0.000 & 0.000 & 0.000 & 0.000 & 0.000 & 0.468 & 0.000 & 0.000 & 0.000 & 0.000 & 0.000 & 0.000 & 0.000 & 0.000 & 0.000 \\
\hline Oman & 0.050 & 0.10 & 0.15 & 0.20 & 0.250 & 0.30 & 0.35 & 0.40 & 0.45 & 0.500 & 0.55 & 0.60 & 0.65 & 0.70 & 0.75 & 0.80 & 0.85 & 0.90 & 0.950 \\
\hline$\Delta \mathrm{ISI}_{\mathrm{t}}$ to $\Delta \mathrm{CBM}_{\mathrm{t}}$ & 0.000 & 0.000 & 0.000 & 0.000 & 0.000 & 0.000 & 0.000 & 0.000 & 0.000 & 0.949 & 0.000 & 0.000 & 0.000 & 0.000 & 0.000 & 0.000 & 0.000 & 0.000 & 0.000 \\
\hline$\Delta \mathrm{CBM}_{\mathrm{t}}$ to $\Delta \mathrm{ISI}_{\mathrm{t}}$ & 0.000 & 0.000 & 0.000 & 0.000 & 0.000 & 0.000 & 0.000 & 0.000 & 0.000 & 0.657 & 0.000 & 0.000 & 0.000 & 0.000 & 0.000 & 0.000 & 0.000 & 0.000 & 0.000 \\
\hline Qatar & 0.050 & 0.10 & 0.15 & 0.20 & 0.250 & 0.30 & 0.35 & 0.40 & 0.45 & 0.500 & 0.55 & 0.60 & 0.65 & 0.70 & 0.75 & 0.80 & 0.85 & 0.90 & 0.950 \\
\hline$\Delta \mathrm{ISI}_{\mathrm{t}}$ to $\Delta \mathrm{CBM}_{\mathrm{t}}$ & 0.000 & 0.000 & 0.000 & 0.000 & 0.000 & 0.000 & 0.000 & 0.000 & 0.000 & 0.042 & 0.000 & 0.000 & 0.000 & 0.000 & 0.000 & 0.000 & 0.000 & 0.000 & 0.000 \\
\hline$\Delta \mathrm{CBM}_{\mathrm{t}}$ to $\Delta \mathrm{ISI}_{\mathrm{t}}$ & 0.000 & 0.000 & 0.000 & 0.000 & 0.000 & 0.000 & 0.000 & 0.000 & 0.000 & 0.801 & 0.000 & 0.000 & 0.000 & 0.000 & 0.000 & 0.000 & 0.000 & 0.000 & 0.000 \\
\hline \multicolumn{20}{|c|}{ Suadi Arabia } \\
\hline Saudi Arabia & 0.050 & 0.10 & 0.15 & 0.20 & 0.250 & 0.30 & 0.35 & 0.40 & 0.45 & 0.500 & 0.55 & 0.60 & 0.65 & 0.70 & 0.75 & 0.80 & 0.85 & 0.90 & 0.950 \\
\hline$\Delta \mathrm{ISI}_{\mathrm{t}}$ to $\Delta \mathrm{CBM}_{\mathrm{t}}$ & 0.000 & 0.000 & 0.000 & 0.000 & 0.000 & 0.000 & 0.000 & 0.000 & 0.000 & 0.236 & 0.000 & 0.000 & 0.000 & 0.000 & 0.000 & 0.000 & 0.000 & 0.000 & 0.000 \\
\hline$\Delta \mathrm{CBM}_{\mathrm{t}}$ to $\Delta \mathrm{ISI}_{\mathrm{t}}$ & 0.759 & 0.000 & 0.000 & 0.000 & 0.000 & 0.000 & 0.000 & 0.000 & 0.000 & 0.000 & 0.000 & 0.000 & 0.000 & 0.000 & 0.000 & 0.000 & 0.000 & 0.000 & 0.065 \\
\hline Turkey & 0.050 & 0.10 & 0.15 & 0.20 & 0.250 & 0.30 & 0.35 & 0.40 & 0.45 & 0.500 & 0.55 & 0.60 & 0.65 & 0.70 & 0.75 & 0.80 & 0.85 & 0.90 & 0.950 \\
\hline$\Delta \mathrm{ISI}_{\mathrm{t}}$ to $\Delta \mathrm{CBM}_{\mathrm{t}}$ & 0.000 & 0.000 & 0.000 & 0.000 & 0.000 & 0.000 & 0.000 & 0.000 & 0.000 & 0.287 & 0.000 & 0.000 & 0.000 & 0.000 & 0.000 & 0.000 & 0.000 & 0.000 & 0.000 \\
\hline$\Delta \mathrm{CBM}_{\mathrm{t}}$ to $\Delta \mathrm{ISI}_{\mathrm{t}}$ & 0.000 & 0.000 & 0.000 & 0.000 & 0.000 & 0.000 & 0.000 & 0.000 & 0.000 & 0.954 & 0.000 & 0.000 & 0.000 & 0.000 & 0.000 & 0.000 & 0.000 & 0.000 & 0.000 \\
\hline UAE & 0.050 & 0.10 & 0.15 & 0.20 & 0.250 & 0.30 & 0.35 & 0.40 & 0.45 & 0.500 & 0.55 & 0.60 & 0.65 & 0.70 & 0.75 & 0.80 & 0.85 & 0.90 & 0.950 \\
\hline$\Delta \mathrm{ISI}_{\mathrm{t}}$ to $\Delta \mathrm{CBM}_{\mathrm{t}}$ & 0.000 & 0.000 & 0.000 & 0.000 & 0.000 & 0.000 & 0.000 & 0.000 & 0.000 & 0.588 & 0.000 & 0.000 & 0.000 & 0.000 & 0.000 & 0.000 & 0.000 & 0.000 & 0.000 \\
\hline$\Delta \mathrm{CBM}_{\mathrm{t}}$ to $\Delta \mathrm{ISI}_{\mathrm{t}}$ & 0.000 & 0.000 & 0.000 & 0.000 & 0.000 & 0.000 & 0.000 & 0.000 & 0.000 & 0.102 & 0.000 & 0.000 & 0.000 & 0.000 & 0.000 & 0.000 & 0.000 & 0.000 & 0.000 \\
\hline
\end{tabular}


Note: $\Delta \mathrm{ISI}_{\mathrm{t}}$ is the log-difference of Islamic stock index; $\triangle \mathrm{CBM}_{\mathrm{t}}$ is the log difference sukuk market.

Table 3(b): Quantile Granger Causality (Sukuk Market and Islamic Stock Index)

\begin{tabular}{|c|c|c|c|c|c|c|c|c|c|c|c|c|c|c|c|c|c|c|c|}
\hline \multicolumn{20}{|c|}{ Bahrain } \\
\hline Bahrain & 0.05 & 0.10 & 0.15 & 0.20 & 0.25 & 0.30 & 0.35 & 0.40 & 0.45 & 0.50 & 0.55 & 0.60 & 0.65 & 0.70 & 0.75 & 0.80 & 0.85 & 0.90 & 0.95 \\
\hline$\Delta \mathrm{ISI}_{\mathrm{t}}$ to $\Delta \mathrm{SM}_{\mathrm{t}}$ & 0.000 & 0.000 & 0.000 & 0.000 & 0.000 & 0.000 & 0.000 & 0.000 & 0.000 & 0.120 & 0.000 & 0.000 & 0.000 & 0.000 & 0.000 & 0.000 & 0.000 & 0.000 & 0.000 \\
\hline$\Delta \mathrm{SM}_{\mathrm{t}}$ to $\Delta \mathrm{ISI}_{\mathrm{t}}$ & 0.000 & 0.000 & 0.000 & 0.000 & 0.000 & 0.000 & 0.000 & 0.000 & 0.000 & 0.759 & 0.000 & 0.000 & 0.000 & 0.000 & 0.000 & 0.000 & 0.000 & 0.000 & 0.000 \\
\hline \multicolumn{20}{|c|}{ Brunei } \\
\hline Brunei & 0.050 & 0.10 & 0.15 & 0.20 & 0.250 & 0.30 & 0.35 & 0.40 & 0.45 & 0.500 & 0.55 & 0.60 & 0.65 & 0.70 & 0.75 & 0.80 & 0.85 & 0.90 & 0.950 \\
\hline$\Delta \mathrm{ISI}_{\mathrm{t}}$ to $\Delta \mathrm{SM}_{\mathrm{t}}$ & 0.528 & 0.000 & 0.000 & 0.000 & 0.000 & 0.000 & 0.000 & 0.000 & 0.000 & 0.009 & 0.000 & 0.000 & 0.000 & 0.000 & 0.000 & 0.000 & 0.000 & 0.000 & 0.000 \\
\hline$\Delta \mathrm{SM}_{\mathrm{t}}$ to $\Delta \mathrm{ISI}_{\mathrm{t}}$ & 0.000 & 0.000 & 0.000 & 0.000 & 0.000 & 0.000 & 0.000 & 0.000 & 0.000 & 0.667 & 0.000 & 0.000 & 0.000 & 0.000 & 0.000 & 0.000 & 0.000 & 0.000 & 0.000 \\
\hline \multicolumn{20}{|c|}{ Kuwait } \\
\hline Kuwait & 0.050 & 0.10 & 0.15 & 0.20 & 0.250 & 0.30 & 0.35 & 0.40 & 0.45 & 0.500 & 0.55 & 0.60 & 0.65 & 0.70 & 0.75 & 0.80 & 0.85 & 0.90 & 0.950 \\
\hline$\Delta \mathrm{ISI}_{\mathrm{t}}$ to $\Delta \mathrm{SM}_{\mathrm{t}}$ & 0.000 & 0.000 & 0.000 & 0.000 & 0.000 & 0.000 & 0.000 & 0.000 & 0.000 & 0.046 & 0.000 & 0.000 & 0.000 & 0.000 & 0.000 & 0.000 & 0.000 & 0.000 & 0.000 \\
\hline$\Delta \mathrm{SM}_{\mathrm{t}}$ to $\Delta \mathrm{ISI}_{\mathrm{t}}$ & 0.000 & 0.000 & 0.000 & 0.000 & 0.000 & 0.000 & 0.000 & 0.000 & 0.000 & 0.931 & 0.000 & 0.000 & 0.000 & 0.000 & 0.000 & 0.000 & 0.000 & 0.000 & 0.000 \\
\hline Libya & 0.050 & 0.10 & 0.15 & 0.20 & 0.250 & 0.30 & 0.35 & 0.40 & 0.45 & 0.500 & 0.55 & 0.60 & 0.65 & 0.70 & 0.75 & 0.80 & 0.85 & 0.90 & 0.950 \\
\hline$\Delta \mathrm{ISI}_{\mathrm{t}}$ to $\Delta \mathrm{SM}_{\mathrm{t}}$ & 0.000 & 0.000 & 0.000 & 0.000 & 0.000 & 0.000 & 0.000 & 0.000 & 0.000 & 0.829 & 0.000 & 0.000 & 0.000 & 0.000 & 0.000 & 0.000 & 0.000 & 0.000 & 0.000 \\
\hline$\Delta \mathrm{SM}_{\mathrm{t}}$ to $\Delta \mathrm{ISI}_{\mathrm{t}}$ & 0.000 & 0.000 & 0.000 & 0.000 & 0.000 & 0.000 & 0.000 & 0.000 & 0.000 & 0.639 & 0.000 & 0.000 & 0.000 & 0.000 & 0.000 & 0.000 & 0.000 & 0.000 & 0.000 \\
\hline \multicolumn{20}{|c|}{ Malaysia } \\
\hline Malaysia & 0.050 & 0.10 & 0.15 & 0.20 & 0.250 & 0.30 & 0.35 & 0.40 & 0.45 & 0.500 & 0.55 & 0.60 & 0.65 & 0.70 & 0.75 & 0.80 & 0.85 & 0.90 & 0.950 \\
\hline$\Delta \mathrm{ISI}_{\mathrm{t}}$ to $\Delta \mathrm{SM}_{\mathrm{t}}$ & 0.000 & 0.000 & 0.000 & 0.000 & 0.000 & 0.000 & 0.000 & 0.000 & 0.000 & 0.255 & 0.000 & 0.000 & 0.000 & 0.000 & 0.000 & 0.000 & 0.000 & 0.000 & 0.000 \\
\hline$\Delta \mathrm{SM}_{\mathrm{t}}$ to $\Delta \mathrm{ISI}_{\mathrm{t}}$ & 0.000 & 0.000 & 0.000 & 0.000 & 0.000 & 0.000 & 0.000 & 0.000 & 0.000 & 0.468 & 0.000 & 0.000 & 0.000 & 0.000 & 0.000 & 0.000 & 0.000 & 0.000 & 0.000 \\
\hline Oman & 0.050 & 0.10 & 0.15 & 0.20 & 0.250 & 0.30 & 0.35 & 0.40 & 0.45 & 0.500 & 0.55 & 0.60 & 0.65 & 0.70 & 0.75 & 0.80 & 0.85 & 0.90 & 0.950 \\
\hline$\Delta \mathrm{ISI}_{\mathrm{t}}$ to $\Delta \mathrm{SM}_{\mathrm{t}}$ & 0.000 & 0.000 & 0.000 & 0.000 & 0.000 & 0.000 & 0.000 & 0.000 & 0.000 & 0.949 & 0.000 & 0.000 & 0.000 & 0.000 & 0.000 & 0.000 & 0.000 & 0.000 & 0.000 \\
\hline$\Delta \mathrm{SM}_{\mathrm{t}}$ to $\Delta \mathrm{ISI}_{\mathrm{t}}$ & 0.000 & 0.000 & 0.000 & 0.000 & 0.000 & 0.000 & 0.000 & 0.000 & 0.000 & 0.657 & 0.000 & 0.000 & 0.000 & 0.000 & 0.000 & 0.000 & 0.000 & 0.000 & 0.000 \\
\hline Qatar & 0.050 & 0.1 & $\mathbf{0}$ & 0.2 & 0.250 & 0.30 & 0.35 & $\mathbf{0 . 4}$ & $\mathbf{0 . 4}$ & 0.500 & 0.55 & 0.60 & 0. & 0.70 & 0.75 & 0.80 & 0.85 & 0.90 & 0.950 \\
\hline$\Delta \mathrm{ISI}_{\mathrm{t}}$ to $\Delta \mathrm{SM}_{\mathrm{t}}$ & 0.000 & 0.000 & 0.000 & 0.000 & 0.000 & 0.000 & 0.000 & 0.000 & 0.000 & 0.042 & 0.000 & 0.000 & 0.000 & 0.000 & 0.000 & 0.000 & 0.000 & 0.000 & 0.000 \\
\hline$\Delta \mathrm{SM}_{\mathrm{t}}$ to $\Delta \mathrm{ISI}_{\mathrm{t}}$ & 0.000 & 0.000 & 0.000 & 0.000 & 0.000 & 0.000 & 0.000 & 0.000 & 0.000 & 0.801 & 0.000 & 0.000 & 0.000 & 0.000 & 0.000 & 0.000 & 0.000 & 0.000 & 0.000 \\
\hline \multicolumn{20}{|c|}{ Suadi Arabia } \\
\hline Saudi Arabia & 0.050 & 0.10 & 0.15 & 0.20 & 0.250 & 0.30 & 0.35 & 0.40 & 0.45 & 0.500 & 0.55 & 0.60 & 0.65 & 0.70 & 0.75 & 0.80 & 0.85 & 0.90 & 0.950 \\
\hline$\Delta \mathrm{ISI}_{\mathrm{t}}$ to $\Delta \mathrm{SM}_{\mathrm{t}}$ & 0.000 & 0.000 & 0.000 & 0.000 & 0.000 & 0.000 & 0.000 & 0.000 & 0.000 & 0.236 & 0.000 & 0.000 & 0.000 & 0.000 & 0.000 & 0.000 & 0.000 & 0.000 & 0.000 \\
\hline$\Delta \mathrm{SM}_{\mathrm{t}}$ to $\Delta \mathrm{ISI}_{\mathrm{t}}$ & 0.759 & 0.000 & 0.000 & 0.000 & 0.000 & 0.000 & 0.000 & 0.000 & 0.000 & 0.000 & 0.000 & 0.000 & 0.000 & 0.000 & 0.000 & 0.000 & 0.000 & 0.000 & 0.065 \\
\hline Turkey & 0.050 & 0.1 & o. & 0.2 & 0.250 & 0. & 0.3 & $\mathbf{0 . 4}$ & $\sigma_{0}$ & 0. & 0.5 & 0 . & $\mathbf{0 .}$ & 0. & 0.' & 0. & 0. & $\mathbf{0 .}$ & 0.950 \\
\hline$\Delta \mathrm{ISI}_{\mathrm{t}}$ to $\Delta \mathrm{SM}_{\mathrm{t}}$ & 0.000 & 0.000 & 0.000 & 0.000 & 0.000 & 0.000 & 0.000 & 0.000 & 0.000 & 0.287 & 0.000 & 0.000 & 0.000 & 0.000 & 0.000 & 0.000 & 0.000 & 0.000 & 0.000 \\
\hline$\Delta \mathrm{SM}_{\mathrm{t}}$ to $\Delta \mathrm{ISI}_{\mathrm{t}}$ & 0.000 & 0.000 & 0.000 & 0.000 & 0.000 & 0.000 & 0.000 & 0.000 & 0.000 & 0.954 & 0.000 & 0.000 & 0.000 & 0.000 & 0.000 & 0.000 & 0.000 & 0.000 & 0.000 \\
\hline UAE & 0.050 & 0.10 & 0.15 & 0.20 & 0.250 & 0.30 & 0.35 & 0.40 & 0.45 & 0.500 & 0.55 & 0.60 & 0.65 & 0.70 & 0.75 & 0.80 & 0.85 & 0.90 & 0.950 \\
\hline$\Delta \mathrm{ISI}_{\mathrm{t}}$ to $\Delta \mathrm{SM}_{\mathrm{t}}$ & 0.000 & 0.000 & 0.000 & 0.000 & 0.000 & 0.000 & 0.000 & 0.000 & 0.000 & 0.588 & 0.000 & 0.000 & 0.000 & 0.000 & 0.000 & 0.000 & 0.000 & 0.000 & 0.000 \\
\hline
\end{tabular}


Note: $\Delta \mathrm{ISI}$ is the log-difference of Islamic stock index; $\Delta \mathrm{SM}_{\mathrm{t}}$ is the log difference sukuk market. 
The study presents quantile granger causality test in Table 3(a) and Table3(b). The findings show that both conventional bond market and Islamic stock index granger cause to each other almost on all quantiles of both variables in all selected countries. Similarly, granger causality between sukuk and Islamic stock markets also indicates that these variables are also granger cause to each other on all quantiles.

\section{Conclusion and Recommendations}

This research explores the asymmetric empirical relationship between sukuk, Conventional bonds and Islamic stock market for the world's top 10 Islamic stock markets using the Quantile-on-Quantile methodology proposed by Sim and Zhou (2015). This methodology provides details on how quantiles of sukuk market and Conventional bonds affect the quantiles of Islamic stock markets and gives information on the inter-linkage between these variables for a more precise and accurate manner. The study employed daily data ranging from $1^{\text {st }}$ January 2008 to $31^{\text {st }}$ December 2019.

\section{Recommendation}

Investment has played an important role in absorbing employment, promoting agricultural development, and increasing tax revenue and has made outstanding contributions to economic growth and social development. The development of stock market can bring information and capital flow. A large number of entrepreneurs, experts and scholars bring the latest technology, information, and advanced management concepts for investment. Islamic stock speeds up the renewal of ideas and promotes the opening of the region and its integration with the international community.

The analysis and the result of the study indicate and provide the evidence regarding the timely and appropriately measurement regarding the correlation changing and the behavior of the conventional bond market, sukuk market and the Islamic stock market in the top Islamic economies of the world which is beneficial for the investors and the policymakers for portfolio diversification and risk management. The portfolio managers can tap our empirical results by combining sukuk, conventional bonds and Islamic stock as assets with asymmetric dependence to hold optimal hedge ratios and portfolio weights in crisis periods and in different market episodes.

However, this study has some limitations, which help to provide a scope for future research in this field. Since, in this study, the data are collected from the top 10 Islamic economies countries to treat the hypothesis, in the future, it may be taken from developing and fast-growing nations and should explore the dynamics of the conventional bond market, sukuk market, bit-coin, mutual fund in those countries with different outcomes. Economies in a particular domain may also be analyzed with investment, such as for residential, commercial, and industrial. 


\section{References}

Ahmed, H., \& Elsayed, A. H. (2019). Are Islamic and conventional capital markets decoupled? Evidence from stock and bonds/sukuk markets in Malaysia. The Quarterly Review of Economics and Finance, 74, 56-66.

Chang, B. H., Sharif, A., Aman, A., Suki, N. M., Salman, A., \& Khan, S. A. R. (2020). The asymmetric effects of oil price on sectoral Islamic stocks: New evidence from quantile-on-quantile regression approach. Resources Policy, 65, 101571.

Coeurdacier, N., Rey, H., \& Winant, P. (2020). Financial integration and growth in a risky world. Journal of Monetary Economics, 112, 1-21.

Duqi, A., \& Al-Tamimi, H. (2019). Factors affecting investors' decision regarding investment in Islamic Sukuk. Qualitative Research in Financial Markets, 11(1), 60-72.

Engle, R. F., \& Granger, C. W. (1987). Co-integration and error correction: representation, estimation, and testing. Econometrica: journal of the Econometric Society, 251-276.

Galvao, A. F. (2009). Essays on quantile regression for dynamic panel data models. University of Illinois at UrbanaChampaign.

Koenker, R., \& Bassett Jr, G. (1978). Regression quantiles. Econometrica: journal of the Econometric Society, 33-50.

Koenker, R., \& Xiao, Z. (2004). Unit root quantile autoregression inference. Journal of the American Statistical Association, 99(467), 775-787.

McMillan, D. G. (2019). Interrelation and Spillover Effects Between Stocks and Bonds: Cross- Market and CrossAsset Evidence. Available at SSRN 3338465.

Meo, M. S., Chowdhury, M. A. F., Shaikh, G. M., Ali, M., \& Masood Sheikh, S. (2018). Asymmetric impact of oil prices, exchange rate, and inflation on tourism demand in Pakistan: new evidence from nonlinear ARDL. Asia Pacific Journal of Tourism Research, 23(4), 408-422.

Naifar, N. (2016). Modeling dependence structure between stock market volatility and sukuk yields: A nonlinear study in the case of Saudi Arabia. Borsa Istanbul Review, 16(3), 157- 166.

Nasir, A., \& Farooq, U. (2017). Analysis of value at risk of Sukuk and conventional bonds in Pakistan. Journal of Islamic Accounting and Business Research.

Shahzad, S. J. H., Bouri, E., Roubaud, D., \& Kristoufek, L. (2020). Safe haven, hedge and diversification for G7 stock markets: Gold versus bitcoin. Economic Modelling, 87, 212- 224.

Shahzad, S. J. H., Raza, N., Shahbaz, M., \& Ali, A. (2017). Dependence of stock markets with gold and bonds under bullish and bearish market states. Resources Policy, 52, 308-319.

Sharif, A., Afshan, S., \& Qureshi, M. A. (2019). Idolization and ramification between globalization and ecological footprints: evidence from quantile-on-quantile Research, 26(11), 11191-11211.

Sharif, A., Mishra, S., Sinha, A., Jiao, Z., Shahbaz, M., \& Afshan, S. (2020). The renewable energy consumptionenvironmental degradation nexus in Top-10 polluted countries: Fresh insights from quantile-on-quantile regression approach. Renewable Energy.

Sharif, A., Mishra, S., Sinha, A., Jiao, Z., Shahbaz, M., \& Afshan, S. (2020). The renewable energy consumptionenvironmental degradation nexus in Top-10 polluted countries: Fresh insights from quantile-on-quantile regression approach. Renewable Energy, 150, 670-690.

Sim, N., \& Zhou, H. (2015). Oil prices, US stock return, and the dependence between their Banking \& Finance, 55, 1-8.

quantiles. Journal of

Xiao, Z. (2009). Quantile cointegrating regression. Journal of econometrics, 150(2), 248-260. 